\title{
Sleep Promotes the Neural Reorganization of Remote Emotional Memory
}

\author{
Virginie Sterpenich, ${ }^{1}$ Geneviève Albouy, ${ }^{1}$ Annabelle Darsaud, ${ }^{1}$ Christina Schmidt, ${ }^{1}$ Gilles Vandewalle, ${ }^{1}$ \\ Thien Thanh Dang Vu, ${ }^{1,3}$ Martin Desseilles, ${ }^{1,4}$ Christophe Phillips, ${ }^{1}$ Christian Degueldre, ${ }^{1}$ Evelyne Balteau, ${ }^{1}$ \\ Fabienne Collette, ${ }^{1,2}$ André Luxen, ${ }^{1}$ and Pierre Maquet $^{1,3}$ \\ ${ }^{1}$ Cyclotron Research Centre and ${ }^{2}$ Department of Cognitive Sciences, University of Liège, and Departments of ${ }^{3}$ Neurology and ${ }^{4}$ Psychiatry, Centre Hospitalier \\ Universitaire du Sart Tilman, B-4000 Liège, Belgium
}

\begin{abstract}
Sleep promotes memory consolidation, a process by which fresh and labile memories are reorganized into stable memories. Emotional memories are usually better remembered than neutral ones, even at long retention delays. In this study, we assessed the influence of sleep during the night after encoding onto the neural correlates of recollection of emotional memories 6 months later. After incidental encoding of emotional and neutral pictures, one-half of the subjects were allowed to sleep, whereas the others were totally sleep deprived, on the first postencoding night. During subsequent retest, functional magnetic resonance imaging sessions taking place $3 \mathrm{~d}$ and $6 \mathrm{months}$ later, subjects made recognition memory judgments about the previously studied and new pictures. Between these retest sessions, all participants slept as usual at home. At 6 month retest, recollection was associated with significantly larger responses in subjects allowed to sleep than in sleep-deprived subjects, in the ventral medial prefrontal cortex (vMPFC) and the precuneus, two areas involved in memory retrieval, as well as in the extended amygdala and the occipital cortex, two regions the response of which was modulated by emotion at encoding. Moreover, the functional connectivity was enhanced between the vMPFC and the precuneus, as well as between the extended amygdala, the vMPFC, and the occipital cortex in the sleep group relative to the sleep-deprived group. These results suggest that sleep during the first postencoding night profoundly influences the long-term systems-level consolidation of emotional memory and modifies the functional segregation and integration associated with recollection in the long term.
\end{abstract}

\section{Introduction}

New memories initially stay in a fragile state and need to be consolidated over time (Müller and Pilzecker, 1900). The mechanisms underlying memory consolidation range from molecular and cellular processes, taking place within minutes or hours after learning, to the progressive reorganization of memory traces across macroscopic brain systems, thought to take place over weeks or months (Dudai, 2004). The classical theory of consolidation proposes that hippocampo-dependent memories become progressively independent of the limbic structures and are gradually stored in cortical circuits (Alvarez and Squire, 1994; Frankland and Bontempi, 2005).

There is evidence that sleep improves memory retention when retrieval is tested hours to days after learning (Maquet, 2001; Born et al., 2006). Potential influence of sleep on long-term retention remains uncertain. A total sleep deprivation (TSD) during the night after the encoding of word pairs hindered the changes in brain responses associated with recall 6 months later, which are otherwise observed if sleep is allowed on the first

Received Feb. 3, 2009; revised Feb. 26, 2009; accepted March 5, 2009.

This work was supported by the Belgian Fonds National de la Recherche Scientifique (FNRS), Fondation Médicale Reine Elisabeth, University of Liège, and Pôles d'Attraction Interuniversitaires Grant P5/04. V.S., A.D., C.S., G.V., T.T.D.V., M.D., F.C., and P.M. are supported by FNRS.

Correspondence should be addressed to Pierre Maquet, Centre de Recherches du Cyclotron, Université de Liège, B30, Sart Tilman, B-4000 Liège, Belgium. E-mail: pmaquet@ulg.ac.be.

DOI:10.1523/JNEUROSCI.0561-09.2009

Copyright $\odot 2009$ Society for Neuroscience $\quad$ 0270-6474/09/295143-10\$15.00/0 postencoding night. Recall after 6 months elicits larger responses in the ventral medial prefrontal cortex (vMPFC) than a recall conducted $48 \mathrm{~h}$ after encoding (Gais et al., 2007).

Emotional memories are better remembered than neutral ones. Recollection of emotional pictures is enhanced, relative to neutral material, even 1 year after encoding, and it is associated with an enhanced activity in the amygdala (Dolcos et al., 2005). Positive effects of sleep on emotional memories have been reported at the behavioral level. Retrieval of emotional (relative to neutral) material several hours after encoding is enhanced if the interval contains an episode of sleep (Hu et al., 2006; Payne et al., 2008; Nishida et al., 2009), especially rapid eye movement (REM) sleep (Wagner et al., 2001), rather than a period of wake. This sleep-dependent advantage persists 4 years later (Wagner et al., 2006).

We recently characterized the effects of sleep on the neural correlates of emotional memory retrieval $72 \mathrm{~h}$ after encoding (Sterpenich et al., 2007). During the recollection of emotional (relative to neutral) items, responses in the hippocampus and the polar medial prefrontal cortex (MPFC) were significantly larger if subjects were allowed to sleep, rather than sleep deprived, on the first postencoding night. These results suggested that sleep favors the systems-level consolidation of emotional memories.

The effects of sleep on the neural correlates of emotional memories have not yet been characterized in the long term. Here, we characterized how sleep influences brain response patterns associated with the recollection of emotional events 6 months 
after encoding. We hypothesized that the differentiation in the neural correlates of memory, detectable $72 \mathrm{~h}$ after encoding between subjects in the sleep and TSD groups, would give rise to divergent longterm consolidation during the following weeks, in such a way that (1) memory for emotional relative to neutral items, would be enhanced in the long term, especially in the sleep group; (2) recollection of remote emotional items would be associated with a reduction of hippocampal responses but with enhanced responses in the vMPFC and the amygdala; and (3) this response pattern would be disrupted by TSD.

\section{Materials and Methods}

Subjects. Participants had no medical, psychiatric, traumatic, or sleep disorders. Their scores on Beck Depression Scale (Steer et al., 1997), Beck Anxiety Scale (Beck et al., 1988), and Bermond-Vorst Alexithymia Questionnaire (Bermond et al., 1999) were within normal range. Extreme morning and evening types, as assessed by the Horne-Ostberg Questionnaire (Horne and Ostberg, 1976), were not included. None complained of excessive daytime sleepiness as assessed by the Epworth Sleepiness Scale (Johns, 1991) and of sleep disturbances as determined by the Pittsburgh Sleep Quality Index Questionnaire (Buysse et al., 1989). All participants were right-handed as indicated by the Edinburgh Inventory (Oldfield, 1971). The self-assessed questionnaires (for depression, anxiety, alexithymia, sleepiness, sleep quality, and circadian rhythms) and age did not revealed any significant difference in both groups $(p>0.5)$.

Forty healthy normally sighted right-handed volunteers gave their written informed consent to take part in this functional magnetic resonance imaging (fMRI) study, which was approved by the Ethics Committee of the Faculty of Medicine of the University of Liège. These volunteers participated in a study on the role of sleep on emotional memory in which they were engaged in a recognition test $72 \mathrm{~h}$ after the exposure to neutral and emotional pictures. One-half of them had been totally deprived of sleep on the first postencoding night. The results dealing with this early retrieval session were already reported (Sterpenich et al., 2007). Six months later, all the participants were contacted and asked to participate to an unannounced retrieval session. Thirty-six subjects eventually accepted to take part in the second leg of the experiment (21 women; mean age and SD, $22.1 \pm 4.1$ years). The sleep group [regular sleep (RS)] consisted of 10 women and 9 men (mean age and SD, $22.3 \pm 3$ ), and the sleep deprivation group (TSD) contained 11 women and 6 men (mean age and SD, $21.8 \pm 4.1$ ). Of these 36 subjects, 3 subjects of the TSD group were discarded from the analysis because the poor level of recognition hindered any meaningful analysis.

Experimental protocol. The experimental design is summarized in Figure 1.

Before the encoding session, participants followed a $7 \mathrm{~d}$ constant sleep schedule as verified by sleep diaries and wrist actigraphy (Actiwatch; Cambridge Neuroscience). During the incidental encoding session that took place between 3:30 and 8:30 P.M., subjects had to rate the valence of 40 negative, 40 positive, and 40 neutral pictures on a 7-point scale $(-3$, very unpleasant; 0 , neutral; +3 , very pleasant). Each picture, extracted from the International Affective Pictorial System (Lang et al., 1999), was displayed for $3 \mathrm{~s}\left(17 \times 23^{\circ}\right)$. Subjects were unaware that their memory for the picture would be subsequently probed. During the postencoding night, one-half of the subjects were totally sleep deprived at the laboratory, under the experimenters' supervision. The other participants went home and slept as usual. After two additional nights, which allowed sleep-deprived participants to recover, subjects performed the first retrieval session [retest after $3 \mathrm{~d}(3 \mathrm{~d}$ retest)] at the same time of day as for the encoding. The 120 previously encoded pictures were randomly mixed with 60 new ones ( 20 negative, 20 positive, and 20 neutral images). Each trial began by a fixation cross $\left(3.75 \times 3.75^{\circ}\right)$ displayed on a light
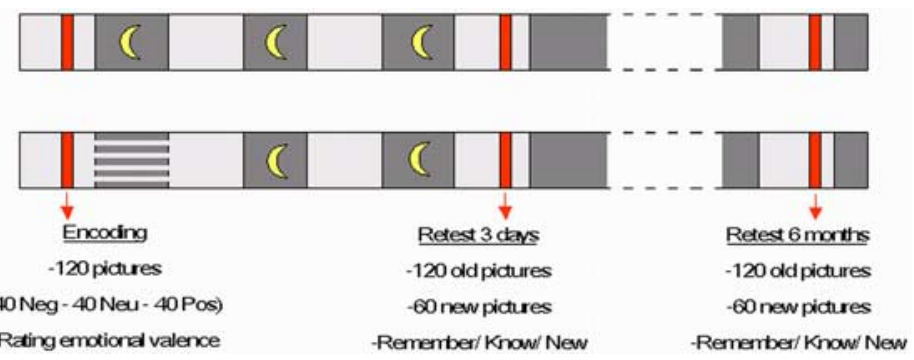

Figure 1. Experimental protocol.

gray background for $1.5 \mathrm{~s}$. A picture was then displayed for $2 \mathrm{~s}\left(17 \times 23^{\circ}\right)$ after which participants had a maximum of $8 \mathrm{~s}$ to perform a remember/ know/new judgment. A "remember" response (R response) indicated that the recognition of the item was associated with retrieval of specific contextual details during encoding (a recollection process). A "know" response (K response) was associated with the feeling of familiarity or the definite feeling of having encoded the item but without being able to retrieve any further specific details. A "new" response was given when the participant thought the item had not been presented during encoding. Participants gave their responses on a three-button keypad, which they held in their right hand. The three first pictures of each session were used for habituation and were modeled separately in the design matrix. Sixty null events, consisting in the presentation of the fixation cross during $6 \mathrm{~s}$, were randomly introduced between the active trials.

Six months after the encoding session (mean \pm SD, 6 months and $13.5 \pm 12.4 \mathrm{~d}$ ), participants were scanned while they performed the second retest session ( 6 month retest), between 2:00 and 7:00 P.M. The 120 initially encoded pictures were again mixed with 60 additional new ones. The new pictures consisted in 20 negative [mean valence on International Affective Picture System (IAPS) and SD, $2.8 \pm 0.6$ ], 20 neutral (mean valence on IAPS and SD, $5.4 \pm 0.6$ ), and 20 positive (mean valence on IAPS, $7.2 \pm 0.3$ ). These pictures contained similar proportion of objects, landscapes, animals, or faces as the encoding ones and the luminance between all pictures ( 180 old plus 60 new) was equalized. Subjects had again to perform a remember/know/new judgment for each stimulus. At the end of the session, outside the scanner, subjects rated the emotional valence of the 60 new pictures with the 7-point scale used during the encoding.

Data acquisition. Data were acquired with a $3 \mathrm{~T}$ head-only magnetic resonance (MR) scanner (Allegra; Siemens) using a gradient echo-planar sequence [echo-planar imaging (EPI), 32 transverse slices with $30 \%$ gap; voxel size, $3.4 \times 3.4 \times 3.4 \mathrm{~mm}$; repetition time $(\mathrm{TR}), 2130 \mathrm{~ms}$; echo time (TE), $40 \mathrm{~ms}$; flip angle, $90^{\circ}$; field of view (FOV), $220 \mathrm{~mm}$ ]. Between 490 and 571 functional volumes were acquired during the $3 \mathrm{~d}$ retest and between 490 and 620 functional volumes during the 6 month retest. In all sessions, the first three volumes were discarded to account for magnetic saturation effects. A structural MR scan was acquired at the end of the experimental session (T1-weighted three-dimensional magnetizationprepared rapid-acquisition gradient echo sequence; TR, $1960 \mathrm{~ms}$; TE, $4.43 \mathrm{~ms}$; inversion time, $1100 \mathrm{~ms}$; FOV, $230 \times 173 \mathrm{~mm}^{2}$; matrix size, $256 \times 192 \times 176$; voxel size, $0.9 \times 0.9 \times 0.9 \mathrm{~mm}$ ). Stimuli were displayed on a screen positioned at the rear of the scanner, which the subject could comfortably see through a mirror mounted on the standard head coil.

Analysis of behavioral data. Memory performances were calculated as the percentage of old items correctly identified as remembered, old items correctly identified as familiar, and new items identified as old (false alarms) during the 6 month retest. In this study, "recognition" is a general term which refers to the capacity of correctly recognizing a previously learned item. We refer to "recollection" as the process of correctly recognizing an item on the basis of the retrieval of specific contextual details ( $\mathrm{R}$ responses). In contrast, familiarity refers to the process of correctly recognizing an item without retrieval of any specific details about the study episode (K responses) (Diana et al., 2007).

Items were split according to the subjective rating of emotion of each subject (negative, score $-3,-2$; neutral, score $-1,0,+1$; and positive, 
score $+2,+3)$. A repeated-measure ANOVA with memory ( $\mathrm{R}$ vs $\mathrm{K})$ and emotion (neg, neu, pos) as within-subjects factors and sleep group (RS vs TSD) as between-subjects factor was performed to test the effect of the group of sleep on memory and emotion separately, the effect of emotion on memory, and the interaction between emotion, memory, and sleep.

To test for a possible confound of a recognition bias because of emotion or sleep on memory performance, we performed an ANOVA on correct rejections, with emotion as within-subject factor and sleep group as between-subjects factor. We calculated also the discrimination index $\left(d^{\prime}\right)$ and the criterion $(C)$ according to the procedure of Snodgrass and Corwin (Snodgrass and Corwin, 1988) for each group of sleep. ANOVAs were also performed to compare groups on the discrimination index and the criterion.

Functional MRI data analysis. In this analysis, we mainly focused on the neural correlates of the $\mathrm{R}$ versus $\mathrm{K}$ responses (i.e., recollection vs familiarity), after 6 months of retention, depending on whether sleep was allowed during the initial postencoding night. Neural correlates of recollection and familiarity are known to substantially differ (Diana et al., 2007). Contrasting R to K responses allows to characterize, among the successful retrieval processes, those that are associated with retrieval of specific contextual details, engage the hippocampus and its related circuits, and are deemed to be reorganized during sleep. In contrast, comparing recollection to "forgotten" responses would confound successful episodic retrieval with unidentified and potentially diverse mechanisms of forgetting (shallow encoding, active forgetting, memory inaccessibility, failure of monitoring...).

The results of the recollection after $3 \mathrm{~d}$ were presented in a previous study (Sterpenich et al., 2007). Nevertheless, the data of the $3 \mathrm{~d}$ retest were also included in the design matrix to allow the measurement of the effect of time (delay) on memory consolidation. Functional MRI data were analyzed using SPM2 (http://www.fil.ion.ucl.ac.uk) implemented in MATLAB 6.5 (Mathworks). Functional scans were realigned using iterative rigid body transformations that minimize the residual sum of square between the first and subsequent images. They were normalized to the MNI EPI template (two-dimensional spline, voxel size, $2 \times 2 \times 2$ $\mathrm{mm}$ ) and spatially smoothed with a Gaussian kernel with full-width at half-maximum (FWHM) of $8 \mathrm{~mm}$.

Data were processed using a two-step analysis, taking into account the intraindividual and interindividual variance, respectively. For each subject, brain responses were modeled at each voxel, using a general linear model. During the both retest session, nine trial types were modeled: old negative, positive, or neutral images correctly recollected during each session (neg-R, pos-R, neu-R); old negative, positive, or neutral images correctly identified as familiar in each session (neg-K, pos-K, neu-K); new negative, positive, or neutral images correctly identified as new (neg-N, pos-N, neu-N). Additional trial types consisted of all forgotten images (misses), false alarms items, and the three first images. Each trial was categorized as neutral or emotional based on the individual subjective ratings. Negative images corresponded to scores -3 and -2 ; neutral images correspond to $-1,0$, and +1 ; and positive images correspond to +2 and +3 . For each trial type, a given item was modeled as a $\delta$ function representing its onset. The ensuing vector was convolved with the canonical hemodynamic response function, and used as a regressor in the individual design matrix. Movement parameters estimated during realignment (translations in $x, y$, and $z$ directions, and rotations around $x$, $y$, and $z$ axes) and a constant vector were also included in the matrix as a variable of no interest. High-pass filter was implemented using a cutoff period of $128 \mathrm{~s}$ to remove the low-frequency drifts from the time series. Serial autocorrelations were estimated with a restricted maximumlikelihood algorithm using an autoregressive model of order 1 (plus white noise). For the 6 month retest, linear contrasts estimated the main effect of emotion (negative vs neutral or positive vs neutral) and memory (remember vs know) for all subjects. However, the contrasts of interest were essentially the emotion by memory interaction [(Neg-R - Neu$\mathrm{R})-(\mathrm{Neg}-\mathrm{K}-\mathrm{Neu}-\mathrm{K})]$ and $[($ Pos-R $-\mathrm{Neu}-\mathrm{R})-($ Pos-K $-\mathrm{Neu}-\mathrm{K})]$. An interaction including the effect of the delay on retention was also applied, comparing the two retrieval sessions per subject: emotion by memory by delay (e.g., 6 month retest $>3 \mathrm{~d}$ retest). The resulting set of voxel values constituted a map of $t$ statistics $[\operatorname{SPM}(\mathrm{T})]$. The individual summary statistical image were spatially smoothed with a Gaussian kernel with FWHM of $6 \mathrm{~mm}$ and used in a second-level analysis, corresponding to a random effect analysis. The second-level analysis consisted in one-sample $t$ tests testing for the effect of interest for both groups together and separately for each group as well as in two-sample $t$ tests comparing the responses between the two groups (RS vs TSD). The latter contrasts corresponded to an emotion by memory by sleep status interaction and to an emotion by memory by sleep status by delay interaction, respectively. The resulting set of voxel values was thresholded at $p<$ 0.001 (uncorrected). Statistical inferences were corrected for multiple comparisons using Gaussian random field theory at the voxel level in a small spherical volume (radius, $10 \mathrm{~mm}$ ) around a priori locations of structures of interest, taken from the literature on declarative memory (supplemental information, available at www.jneurosci.org as supplemental material). This published information collected in independent samples on similar tasks constitutes a spatial prior information that usefully constrains statistical inferences.

When useful, exclusive masking was used to isolate responses specific to the RS group. A mask was created by thresholding a given contrast map in the TSD group at a very lenient threshold $(p=0.5)$ and excluding these voxels from the corresponding contrast map of the RS group.

Results concerning positive items were analyzed in the same way and are presented in supplemental Results (available at www.jneurosci.org as supplemental material).

Bayesian inferences and posterior probability maps. In classical statistics, nonsignificant results (i.e., not rejecting the null hypothesis of no difference in response between conditions) do not guarantee an absence of effect. To check that the hippocampus was not activated during retrieval 6 months after encoding, we quantified the probability of response in the hippocampus, by computing posterior probability maps that enable conditional or Bayesian inferences about regionally specific effects (Friston and Penny, 2003). Posterior probability maps and effect size were computed for the contrast of the interaction between memory and delay for all subjects to verify that the hippocampus was not activated during recollection of remote emotional memory.

Psychophysiological interaction analyses. Psychophysiological interaction (PPI) analyses were computed to test the hypothesis that functional connectivity between seed regions (the amygdala and the ventral medial prefrontal cortex) (see Results) and the rest of the whole brain not only differed between emotional and neutral recognized items but were also influenced by the sleep status during the first postencoding night (RS or TSD). For each individual, the coordinates of the seed areas corresponded to the local maxima detected within $6 \mathrm{~mm}$ of the peak voxel of the RFX analysis.

A new linear model was prepared for PPI analyses at the individual level, using three regressors. One regressor represented the emotional status of recollected pictures (Neg-R vs Neu-R) or (Pos-R vs Neu-R). The second regressor was the activity in the reference areas. The third regressor represented the interaction of interest between the first (psychological) and the second (physiological) regressors. To build this regressor, the underlying neuronal activity was first estimated by a parametric empirical Bayes formulation, combined with the psychological factor and subsequently convolved with the hemodynamic response function (Gitelman et al., 2003). The model also included movement parameters. A significant psychophysiological interaction indicated a change in the regression coefficients between any reported brain area and the reference region, related the retrieval of emotional (rather than neutral) stimuli correctly recollected. Next, individual summary statistic images obtained at the first-level (fixed-effects) analysis were spatially smoothed $(6 \mathrm{~mm}$ FWHM Gaussian kernel) and entered in a second-level (random-effects) analysis using two-sample $t$ tests to compare the functional connectivity between groups. Inferences were conducted as for the main effect analysis.

\section{Results}

\section{Sleep parameters}

Sleep duration and quality were assessed by questionnaires. Mean subjective sleep duration and quality were not significantly different between groups on the night preceding the session re- 


\begin{tabular}{|c|c|c|c|c|c|c|}
\hline & \multicolumn{6}{|c|}{ Memory performance (\%) } \\
\hline & \multicolumn{3}{|l|}{ Sleep group } & \multicolumn{3}{|l|}{ TSD group } \\
\hline & Negative & Neutral & Positive & Negative & Neutral & Positive \\
\hline Remember (R) & $30.2 \pm 20.1$ & $19.0 \pm 15.0$ & $27.6 \pm 18.5$ & $26.9 \pm 13.3$ & $13.4 \pm 10.0$ & $19.6 \pm 12.7$ \\
\hline Know (K) & $31.2 \pm 9.0$ & $29.8 \pm 8.2$ & $30.4 \pm 12.5$ & $32.2 \pm 13.9$ & $33.2 \pm 9.9$ & $31.5 \pm 14.9$ \\
\hline Correct rejection $(\mathrm{Cr})$ & $83.6 \pm 14.4$ & $85.7 \pm 13.0$ & $90.0 \pm 13.7$ & $93.7 \pm 9.3$ & $92.0 \pm 5.6$ & $90.9 \pm 12.9$ \\
\hline
\end{tabular}

corded after 6 months (mean duration \pm SD: RS, $8.8 \pm 1.3 \mathrm{~h}$; TSD, $8.1 \pm 1.3 \mathrm{~h} ; t_{(34)}$ $=1.6 ; p=0.11$ ) (mean quality on a $1-6$ point scale in which 6 is a very good night \pm SD: RS, $5.0 \pm 0.9$; TSD , $4.9 \pm 0.7$; $\left.t_{(34)}=0.44 ; p=0.66\right)$. At debriefing, there was no evidence for difference in sleep quality in the last 6 months between groups, and in particular, Pittsburgh Sleep Quality Index score was equivalent between groups (mean $\pm \mathrm{SD}$ : RS, $3.5 \pm 1.7$; $\left.\mathrm{TSD}, 2.9 \pm 1.6 ; t_{(34)}=1.08 ; p=0.29\right)$.

\section{Behavioral results}

The proportion of correctly recollected negative, neutral, and positive items was consistently smaller after sleep deprivation, relative to the sleep condition (Neg-R: RS > TSD, $30.2>$ 26.9\%; Neu-R: RS > TSD, $19.0>13.4 \%$; Pos-R: RS $>$ TSD, $27.6>19.6 \%$ ) (Table 1, Fig. 2). However, these differences were not significant because of a large variability in recollection rate between subjects $(p>0.1)$. An ANOVA on memory performance at 6 months with memory type [remember (R) vs know $(\mathrm{K})$ ] and emotion (Neg vs Neu vs Pos) as within-subjects factors and sleep group (RS vs TSD) as between-subjects factor showed no significant main effect of sleep on all hits $(\mathrm{R}+\mathrm{K}$ responses; $\left.F_{(1,30)}=0.56 ; p=0.46\right)$. We observed a significant main effect of emotion $\left(F_{(2,60)}=11.5 ; p<0.001\right)$ because emotional items were better recognized than neutral ones, but no emotion by sleep condition interaction $\left(F_{(2,60)}=0.52 ; p=0.60\right)$. There was a main effect of memory type ( $\mathrm{R}$ vs $\mathrm{K}$ responses; $F_{(1,30)}=6.63 ; p<0.01$ ) because there was more familiarity responses than recollections but no significant interaction between memory and sleep status $\left(F_{(1,30)}=1.25 ; p=0.27\right)$. The emotion by memory type interaction was significant $\left(F_{(2,60)}=6.8 ; p=0.002\right)$. Only recollected items carried out the effect of emotion. Both negative and positive pictures were better recollected than neutral ones (planned comparisons, Neg-R $>$ Neu-R, $p<0.001$; Pos-R $>$ Neu-R, $p<$ $0.001)$. Negative pictures tended to be better recollected than positive ones $(p=0.08)$. In contrast, the percentages of know responses were not affected by emotion (Neg-K $<$ Neu-K, $p=$ 0.92; Pos-K $<$ Neu-K, $p=0.77$; Neg-K $>$ Pos-K, $p=0.73$ ) (Table $1)$. The triple interaction between emotion, memory, and sleep was not significant $\left(F_{(2,60)}=0.35 ; p=0.71\right)$ : emotional events were not differently recognized than neutral ones after sleep or sleep deprivation 6 months later.

An ANOVA computed on correct rejections (new pictures identified as new) showed no effect of sleep $\left(F_{(1,30)}=2.9 ; p=\right.$ $0.10)$, no effect of emotion $\left(F_{(2,60)}=0.38 ; p=0.68\right)$, and no significant emotion by sleep interaction $\left(F_{(2,60)}=1.88 ; p=0.16\right)$. Two other ANOVA on the discrimination index $\left(d^{\prime}\right)$ and the criterion (C) (Snodgrass and Corwin, 1988) showed no signifi-

cant difference between groups $\left(d^{\prime}, F_{(1,30)}=3.31, p>0.05 ; C\right.$, $\left.F_{(1,30)}=3.31, p>0.05\right)$. These results show that there was no response bias because of emotion or sleep.

Finally, an ANOVA computed on memory performances at $3 \mathrm{~d}$ and at 6 months showed a significant effect of time $\left(F_{(1,30)}=\right.$ 184.4; $p<0.001)$ because performance decreases for remote memories and a significant interaction between memory and time $\left(F_{(1,30)}=47.5 ; p<0.001\right)$, because only recollected responses were altered by time ( 6 month retest vs 3 d retest: $R$ responses, $F_{(1,30)}=109.5, p<0.001 ; \mathrm{K}$ responses, $F_{(1,30)}=$ $1.89, p=0.18)$. The interaction between group and time was not significant $\left(F_{(1,30)}=0.1 ; p=0.92\right)$, suggesting that the decrease in performance because of time was equivalent in both groups of subjects.

In summary, the behavioral results showed that the recollection rate decreases with time (from 73 to $25 \%$ ), but emotional items (negative and positive) are still better recollected than neutral ones even 6 months after the encoding session. Moreover, we observed a decrease in performances for negative, neutral, and positive recollected items after sleep deprivation, but it was not significant because of a large variability between subjects.

\section{Functional MRI results}

The main effects of emotion ( $\mathrm{Neg}>\mathrm{Neu}$ and $\mathrm{Pos}>\mathrm{Neu}$ ) was consistent with previous reports in the literature (Sterpenich et al., 2007) and is presented in the supplemental data (supplemental Table S1, available at www.jneurosci.org as supplemental material). As our predictions concern the effects of sleep on explicit memory consolidation, we contrasted recollection (R responses) and familiarity (K responses), because it probes the memory processes that precisely contribute to explicit retrieval. The contrasts will be presented from simple main effects to more complex interactions with two, three, and four factors. We first present the main effect of memory, independently of emotion, and its interaction with sleep and delay separately. We next focus on the emotion by memory interaction, during the 6 month retest ses- 

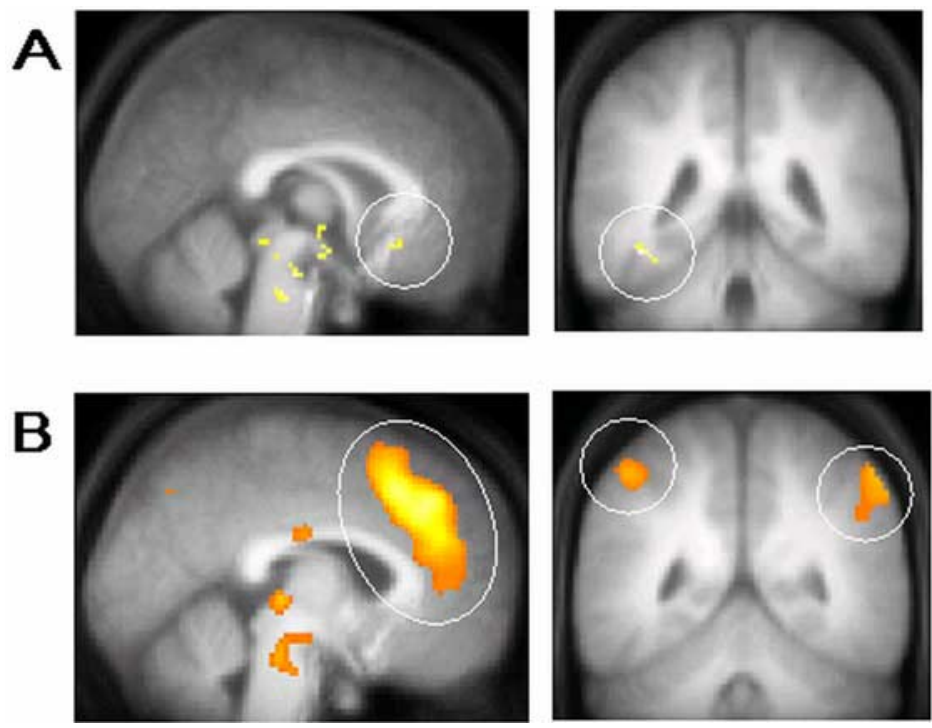

Figure 3. Effect of delay and sleep on memory, independently of emotion. $A$, Effect of sleep (RS $>$ TSD) on memory (R $>K$ ) 6 months after encoding. The effect of memory for the RS group was exclusively masked by the effect of memory for the TSD group. The vMPFC and the fusiform gyrus are displayed on the mean structural MR image, at $p<0.001$ uncorrected. $\boldsymbol{B}$, Effect of delay (6 month retest $>3 \mathrm{~d}$ retest) on memory ( $\mathrm{R}>\mathrm{K}$ ). Remote memory showed significant response in the MPFC and parietal cortices compared with recent memory (display at $p<0.001$ uncorrected).

sion to specify the effect of emotion on remote memory. Subsequently, we will present the three-factor interaction between emotion, memory, and sleep (comparison between RS and TSD group), and finally, we will present the four-factor interaction, presenting the role of sleep in the consolidation of long-term (compared with short-term, between-session comparison) emotional memory.

\section{Effect of sleep and delay on memory, regardless of the emotional significance of stimuli}

To link the present work with previous studies of long-term memory consolidation (Takashima et al., 2006; Gais et al., 2007), we detail here the effect of sleep and delay on memory. The memory contrast $(\mathrm{R}>\mathrm{K})$ during the 6 month retest showed, among others, responses in a large frontoparietal network including the medial prefrontal cortex and in the hippocampus (supplemental Table S2, available at www.jneurosci.org as supplemental material). Interestingly, the between-group comparison (RS vs TSD) for this contrast did not show any significant difference. However, we identified several brain areas that were significantly recruited during remember, relative to know, responses, in the RS group but not in the TSD group (exclusive mask thresholded at $p=0.05)$ (Fig. $3 A$ ). We observed a significant response in the $\operatorname{vMPFC}\left(x, y, z, \mathrm{~mm}: 0,24,-12 ; Z\right.$-score, $\left.3.45 ; p_{\mathrm{svc}}, 0.021\right)$, the fusiform gyrus $\left(x, y, z, \mathrm{~mm}\right.$ : $-38,-44,-18 ; Z$-score, $4.0 ; p_{\mathrm{svc}}$, $0.004)$, and the precuneus $(x, y, z, \mathrm{~mm}:-8,-66,40 ; Z$-score, $3.43 ; p_{\text {svc }}, 0.023$ ) among other regions, suggesting that sleep modified to some extent the recollection of remote memory.

We next examined responses associated with recollection $(\mathrm{R}>\mathrm{K})$ that changed over time, namely between 6 month and $3 \mathrm{~d}$ retests. We compared the remote versus recent retest over all subjects because we did not observed any significant differences between groups $[(\mathrm{R}>\mathrm{K}) \times(6$ month retest $>3 \mathrm{~d}$ retest $)]$ (Fig. $3 B$, Table 2). Significant responses were found in cortical areas including the medial and lateral prefrontal cortex, the inferior parietal cortex, the precuneus, and the putamen. Interestingly, we found no significant responses associated with recollection in the
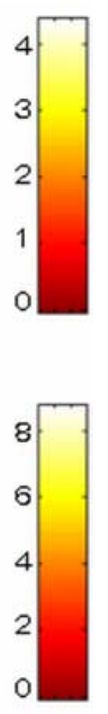

hippocampus. The posterior probability of response in the hippocampus, as inferred by Bayesian statistics, was null, suggesting that this region was not significantly activated in this contrast. In contrast, the reverse interaction, comparing regions more activated for recent that remote memory $[(\mathrm{R}>\mathrm{K}) \times(3 \mathrm{~d}$ retest $>$ 6 month retest)] did show a significant response in the hippocampus during recollection (Table 2), suggesting that the hippocampus is recruited during early but not remote recollection.

Finally, we performed a multiple regression analysis for the contrast $(\mathrm{R}>\mathrm{K})$ of the RS group with the percentage of recollections during the 6 month retest session. We observed a significant regression in the MPFC $(x, y, z, \mathrm{~mm}: 16,50,-4$; $Z$-score, $\left.3.57 ; p_{\text {svc }}, 0.027\right)$, suggesting that the recruitment of this cortical area is proportional to the recollection rate of remote memories. Collectively, these results are in keeping with a progressive engagement of cortical areas and a disengagement of the hippocampus during recollection of remote compared with recent memory, independently of emotion and whether or not sleep was allowed on the first postencoding night.

\section{Effect of sleep and delay on emotional memory}

More specific to the focus of the present study is the interaction between emotion and memory after 6 months of retention [ $(\mathrm{R}>$ $\mathrm{K}) \times(\mathrm{Neg}>\mathrm{Neu})]($ Table 3$)$. We here detail this interaction contrast for negative (compared with neutral) items. We observed similar results with positive pictures but at lower statistical levels (supplemental Table S3, available at www.jneurosci.org as supplemental material). The subjects allowed to sleep significantly recruited the frontal cortex (including the vMPFC), the parietal cortex (precuneus), the occipital lobe (fusiform gyrus), and limbic areas including the amygdala and the hippocampus. The sleep-deprived subjects only engaged the ventral medial prefrontal cortex.

The between-group comparison, corresponding to an emotion by memory by sleep status interaction (Table 4, Fig. 4A) (for positive emotion, see supplemental Table S4, available at www. jneurosci.org as supplemental material), identified significant differences in brain responses. Responses induced by recollection were significantly larger in RS group than in TSD group in the frontal cortex (including the vMPFC and the lateral middle frontal sulcus), the parietal cortex (including the precuneus and the superior parietal gyrus), the middle occipital cortex, and the amygdala. As shown by the activity estimates (Fig. $4 A$ ), in the RS group, the response in these regions was larger for negative than neutral pictures, and more so for the recollected than known items, whereas the reverse pattern was observed in the TSD group. We observed no significantly larger response for the sleepdeprived subjects compared with subjects allowed to sleep.

These regions potentially belong to two different functional networks. The frontoparietal network is classically involved in memory recollection (Fletcher et al., 1997; Henson et al., 2005; Daselaar et al., 2006). In contrast, the amygdalo-occipital network is typically involved in emotional processing (Lane et al., 1999; Pessoa et al., 2002; Vuilleumier et al., 2003; Adolphs, 2004). 
Table 2. Interaction between memory $(\mathrm{R}>\mathrm{K})$ and delay

\begin{tabular}{|c|c|c|c|c|c|}
\hline & Brain regions & MNI coordinates $(x, y, z)$ (in mm) & Z-score & Side & $p_{\text {svc }}$ \\
\hline \multicolumn{6}{|c|}{$\mathrm{RS}+\mathrm{TSD}$ groups (one-sample $t$ test) ( 6 month retest $>3 \mathrm{~d}$ retest) } \\
\hline \multirow[t]{7}{*}{ Frontal cortex } & Superior medial prefrontal gyrus & $4,36,42$ & 5.56 & $\mathrm{R}$ & $0.001^{*}$ \\
\hline & & $6,24,50$ & 5.38 & $\mathrm{R}$ & $0.004^{*}$ \\
\hline & Ventral medial prefrontal gyrus & $6,38,6$ & 3.73 & $\mathrm{R}$ & 0.009 \\
\hline & Lateral middle prefrontal gyrus & $34,50,8$ & 5.38 & $\mathrm{R}$ & $0.027^{*}$ \\
\hline & & $-32,52,2$ & 4.24 & $\mathrm{~L}$ & 0.002 \\
\hline & Lateral inferior prefrontal cortex & $38,20,-12$ & 5.87 & $\mathrm{R}$ & $<0.001^{*}$ \\
\hline & & $-40,18,-14$ & 5.51 & $\mathrm{~L}$ & $0.002^{*}$ \\
\hline \multirow[t]{3}{*}{ Parietal cortex } & Inferior parietal gyrus & $54,-54,52$ & 4.01 & $\mathrm{R}$ & 0.004 \\
\hline & & $-48,-50,52$ & 3.76 & $\mathrm{~L}$ & 0.008 \\
\hline & Precuneus & $2,-74,48$ & 3.19 & $\mathrm{R}$ & 0.043 \\
\hline Basal ganglia & Putamen & $14,12,0$ & 5.13 & $\mathrm{R}$ & $0.011^{*}$ \\
\hline \multicolumn{6}{|c|}{$\mathrm{RS}+\mathrm{TSD}$ groups (one-sample $t$ test) ( $3 \mathrm{~d}$ retest $>6$ month retest) } \\
\hline Limbic area & Hippocampus & $38,-30,-8$ & 3.29 & $\mathrm{R}$ & 0.033 \\
\hline
\end{tabular}

All of the regions presented are thresholded at $p<0.001$ (uncorrected) and significant at $p<0.05$ after correction on a small volume of interest, excepted for the following: ${ }^{*}$ significant at $p<0.05$ corrected over the entire brain volume $\left[p_{\text {svc }} p\right.$ value after small volume correction on coordinates found in the literature referenced in supplemental data (available at www.jneurosci.org as supplemental material)].

Table 3. Interaction between emotion and memory (Neg $>$ Neu $) \times(R>K)$ after 6 months

\begin{tabular}{|c|c|c|c|c|c|}
\hline & Brain regions & MNI coordinates $(x, y, z)$ (in mm) & Z-score & Side & $p_{\text {svc }}$ \\
\hline \multicolumn{6}{|c|}{$\mathrm{RS}$ group (one-sample $t$ test) } \\
\hline \multirow[t]{3}{*}{ Frontal cortex } & Medial prefrontal cortex & $-14,50,4$ & 3.42 & $\mathrm{~L}$ & 0.033 \\
\hline & Ventral medial prefrontal cortex & $-8,34,-6$ & 3.33 & $\mathrm{~L}$ & 0.041 \\
\hline & & $16,30,-8$ & 3.28 & $\mathrm{R}$ & 0.046 \\
\hline Parietal cortex & Precuneus & $-2,-64,64$ & 3.31 & $\mathrm{~L}$ & 0.044 \\
\hline Occipital cortex & Fusiform gyrus & $-34,-46,-22$ & 3.15 & $\mathrm{~L}$ & 0.039 \\
\hline \multirow[t]{2}{*}{ Limbic areas } & Hippocampus & $-40,-32,-6$ & 0.02 & $\mathrm{~L}$ & 3.59 \\
\hline & Amygdala & $24,-8,-18$ & 3.14 & $\mathrm{R}$ & 0.040 \\
\hline \multicolumn{6}{|c|}{ TSD group (one-sample $t$ test) } \\
\hline Frontal cortex & Ventral medial prefrontal cortex & $-4,24,-4$ & 3.12 & $\mathrm{~L}$ & 0.050 \\
\hline
\end{tabular}

Table 4. Interaction between emotion ( $N e g>$ Neu), memory $(R>K)$, and sleep status (RS > TSD) after 6 months

\begin{tabular}{|c|c|c|c|c|c|}
\hline $\mathrm{RS}>\mathrm{TSD}$ groups (two-sample $t$ test) & Brain regions & MNI coordinates $(x, y, z)$ (in $\mathrm{mm}$ ) & Z-score & Side & $p_{\text {svc }}$ \\
\hline \multirow[t]{7}{*}{ Frontal cortex } & \multirow[t]{3}{*}{ Medial prefrontal cortex } & $-8,60,-4$ & 3.33 & $\mathrm{~L}$ & 0.031 \\
\hline & & $0,64,16$ & 3.21 & $\mathrm{R}$ & 0.044 \\
\hline & & $8,40,2$ & 3.19 & $\mathrm{R}$ & 0.047 \\
\hline & \multirow[t]{2}{*}{ Ventral medial prefrontal cortex } & $-12,32,-8$ & 3.14 & $\mathrm{~L}$ & 0.032 \\
\hline & & $8,40,2$ & 3.19 & $\mathrm{R}$ & 0.047 \\
\hline & \multirow[t]{2}{*}{ Lateral middle frontal sulcus } & $26,14,44$ & 4.48 & $\mathrm{R}$ & 0.001 \\
\hline & & $-50,24,34$ & 3.60 & $\mathrm{~L}$ & 0.015 \\
\hline \multirow[t]{4}{*}{ Parietal cortex } & Precuneus & $-4,-66,52$ & 3.72 & $\mathrm{~L}$ & 0.01 \\
\hline & \multirow[t]{3}{*}{ Superior parietal gyrus } & $22,-62,56$ & 3.32 & $\mathrm{R}$ & 0.033 \\
\hline & & $-28,-54,58$ & 3.26 & $\mathrm{~L}$ & 0.038 \\
\hline & & $32,-60,54$ & 3.21 & $\mathrm{R}$ & 0.044 \\
\hline Occipital cortex & Middle occipital gyrus & $-46,-70,-4$ & 4.38 & $\mathrm{~L}$ & 0.001 \\
\hline \multirow[t]{2}{*}{ Limbic areas } & \multirow[t]{2}{*}{ Amygdala } & $26,-6,-16$ & 3.71 & $\mathrm{R}$ & 0.010 \\
\hline & & $34,-4,-36$ & 3.22 & $\mathrm{R}$ & 0.042 \\
\hline
\end{tabular}

Our data further confirmed this functional segregation. First, we inclusively masked the interaction contrast $[(\mathrm{Neg}>\mathrm{Neu}) \times$ $(\mathrm{R}>\mathrm{K}) \times(\mathrm{RS}>\mathrm{TSD})]$ during the 6 month retest by timedependent changes in memory-related responses $[(\mathrm{R}>\mathrm{K}) \times(6$ month retest $>3 \mathrm{~d}$ retest)]. We observed that only frontal (including the vMPFC and the lateral middle frontal gyrus) and parietal cortices (including the precuneus and the superior parietal gyrus) were common to both contrasts, suggesting that these areas are related to the changes in brain response patterns underpinning long-term memory recollection. We also inclusively masked the interaction contrast by the effect of emotion (Neg $>$ $\mathrm{Neu}$ ) during the encoding session because this contrast corresponds to the first emotional processing of the pictures. We ob- served that only the amygdala, the medial prefrontal cortex, and the middle occipital cortex were common to the two contrasts, suggesting that these regions are involved in the processing of emotion. The two amygdalar clusters found in this contrast might correspond to the central and lateral nuclear complexes, which correspond to the output and input structures of the amygdala, respectively. These two areas also differ from the amygdalar cluster detected in the interaction between emotion and memory during the $3 \mathrm{~d}$ retest in the TSD group (Sterpenich et al., 2007) (supplemental Fig. S1, available at www.jneurosci.org as supplemental material), suggesting that different nuclei of the amygdala are involved in the early and late stage of emotional memory consolidation. 

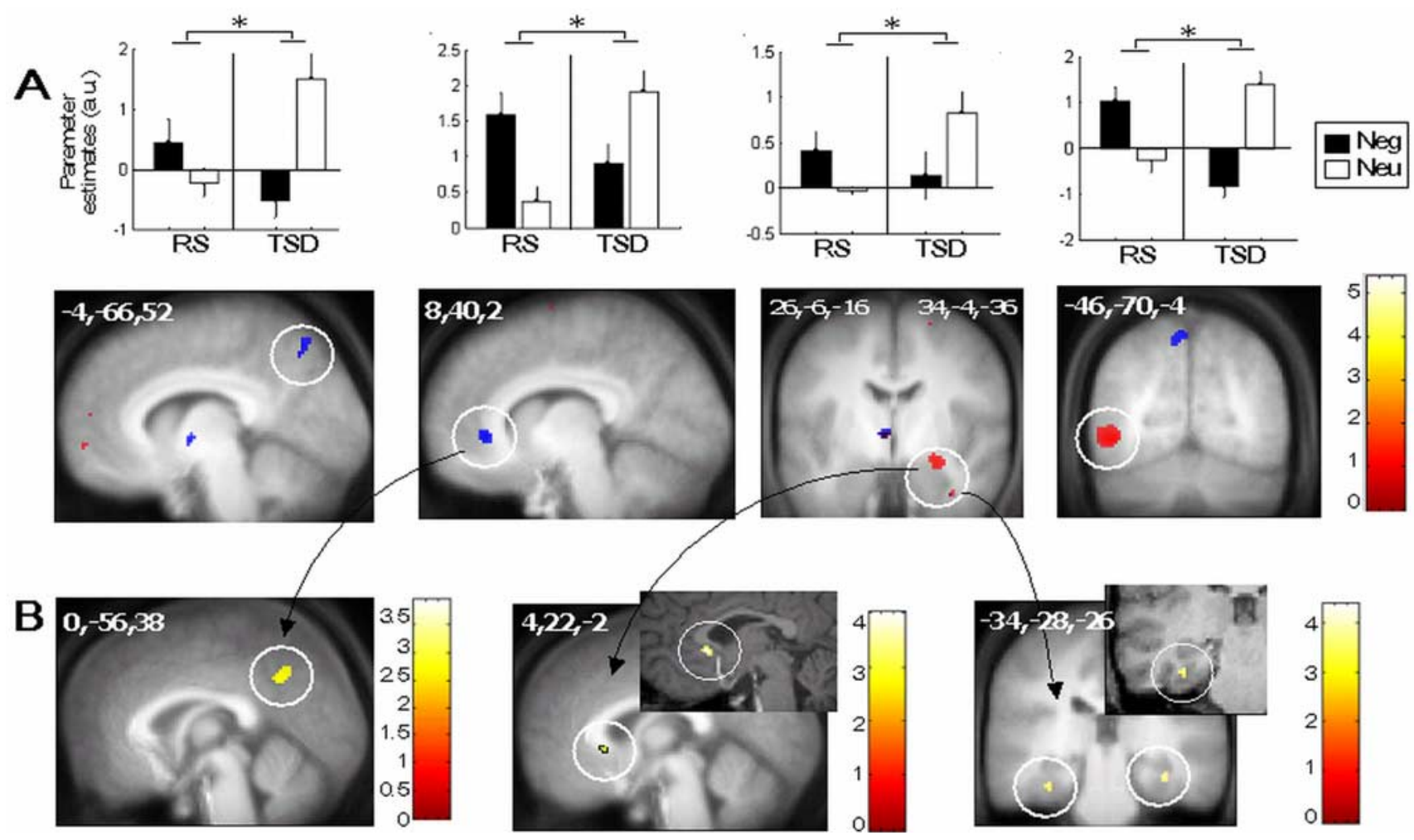

Figure 4. A, Effect of sleep on emotional memory after 6 months. Memory ( $\mathrm{R}>\mathrm{K}$ ) by emotion (Neg $>$ Neu) by sleep status (RS $>$ TSD) interaction. From the left to the right are the following: superior parietal gyrus, vMPFC, amygdala, and middle occipital gyrus. Regions in red are common to the effect of emotion during encoding, and regions in blue are common to the memory by delay interaction. Graphics represent the activity estimates of recollected versus know responses (arbitrary units \pm SEM). Statistical bars only demonstrated the significant differences between groups $\left({ }^{*}\right.$ significant at $\left.p<0.05\right)$. B, PPI. Regions more connected to seed areas (vMPFC and amygdala found in the previous interaction) for negative than neutral correctly recollected pictures and more so in RS than TSD group. From the left to the right are the following: precuneus, VMPFC, and bilateral fusiform gyri. Functional results are displayed on the mean structural MR image (inset, enlarged prefrontal and temporal region in a representative subject).

Table 5. Psychophysiological interactions

\begin{tabular}{|c|c|c|c|c|c|}
\hline RS $>$ TSD groups (two-sample $t$ test) & Brain regions & MNI coordinates $(x, y, z)$ (in $\mathrm{mm}$ ) & Z-score & Side & $p_{\text {svc }}$ \\
\hline VMPFC & Precuneus & $0,-56,38$ & 3.35 & $\mathrm{~L}$ & 0.027 \\
\hline \multirow[t]{2}{*}{ Central amygdala } & Collateral sulcus/fusiform gyrus & $-34,-28,-26$ & 3.32 & $\mathrm{~L}$ & 0.047 \\
\hline & & $36,-30,-20$ & 3.35 & $\mathrm{R}$ & 0.034 \\
\hline Lateral amygdala & VMPFC & $4,22,-2$ & 3.56 & $\mathrm{R}$ & 0.014 \\
\hline
\end{tabular}

We also assessed the functional connectivity of two reference regions during the 6 month retest, testing whether they would establish distinct functional connections with other brain areas, in the context of emotional compared with neutral hits, and depending on whether subjects were allowed to sleep during the postencoding night. We chose two seed regions in the interaction between emotion $(\mathrm{Neg}>\mathrm{Neu})$, memory $(\mathrm{R}>\mathrm{K})$, and sleep (RS $>$ TSD) described above: the vMPFC (involved in long-term memory) and the amygdala (involved in emotional processing). The vMPFC was functionally connected to the precuneus; the lateral part of the amygdala was connected to the vMPFC; the dorsal part of the amygdala was connected bilaterally to anterior portion of the fusiform gyrus, more in RS than in TSD group and more for negative than neutral hits (Table 5, Fig. $4 B$ ) (for positive emotion, see supplemental Table S5, available at www.jneurosci. org as supplemental material). In the orbitofrontal region, susceptibility artifacts can cause some spatial distortion, which potentially explains the posterior location of vMPFC responses close to the subgenual corpus callosum observed in this contrast.

Interestingly, there were no significant changes in responses during recollection of negative, relative to neutral items, between 6 month and 3 d retest sessions, regardless of the sleep status [emotion by memory by delay interaction: $(\mathrm{Neg}>\mathrm{Neu}) \times(\mathrm{R}>\mathrm{K}) \times(6$ month retest $>3$ d retest $)]$. This finding is attributable to the fact that there was no change in response between $3 \mathrm{~d}$ and 6 month retest in the sleep-deprived subjects. In contrast, in the RS group, this interaction was significant in the amygdala $(x, y, z, \mathrm{~mm}: 32,-4,-34$; $Z$-score $\left.=3.66 ; p_{\mathrm{svc}}=0.017\right)$ and in the medial prefrontal cortex $\left(x, y, z\right.$, mm: $16,54,4 ; Z$-score $\left.=3.19 ; p_{\mathrm{svc}}=0.047\right)$.

Finally, we assessed whether recollection-related responses to negative, relative to neutral, items would differ between groups and more so after 6 months than after $3 \mathrm{~d}$. This contrast corresponds to an emotion by memory by sleep status by delay interaction $[(\mathrm{Neg}>\mathrm{Neu}) \times(\mathrm{R}>\mathrm{K}) \times(\mathrm{RS}>\mathrm{TSD}) \times(6$ month retest $>3 \mathrm{~d}$ retest) $]$. We observed a significantly larger response in the frontal cortex (including the vMPFC and the lateral superior frontal sulcus), in the middle occipital cortex, and in the amygdala (Table 6, Fig. 5) (for positive emotion, see supplemental Table S6, available at www.jneurosci.org as supplemental material). Activity estimates show that recollection of negative, relative to neutral, items elicit larger responses after 6 months than 
Table 6. Interaction between emotion (Neg $>$ Neu), memory (R > K), sleep status (RS > TSD), and delay (6 months $>3 \mathrm{~d}$ )

\begin{tabular}{|c|c|c|c|c|c|}
\hline $\begin{array}{l}\text { RS }>\text { TSD groups (two-sample } t \text { test), } \\
\quad 6 \text { months }>3 \mathrm{~d}\end{array}$ & Brain regions & MNI coordinates $(x, y, z)$ (in mm) & Z-score & Side & $p_{\text {svc }}$ \\
\hline \multirow[t]{2}{*}{ Frontal cortex } & Ventral medial prefrontal cortex & $12,30,-10$ & 3.15 & $\mathrm{R}$ & 0.049 \\
\hline & Lateral superior frontal sulcus & $-48,-2,34$ & 3.40 & $\mathrm{~L}$ & 0.025 \\
\hline Occipital cortex & Middle occipital gyrus & $-50,-68,-6$ & 3.76 & $\mathrm{~L}$ & 0.008 \\
\hline Limbic area & Amygdala & $34,-6,-38$ & 4.20 & $\mathrm{R}$ & 0.002 \\
\hline
\end{tabular}

after $3 \mathrm{~d}$ and more so in RS than TSD group. We observed no significant response for the TSD group compared with the RS group.

\section{Discussion}

In this study, brain responses associated with successful recollection of emotional and neutral pictures were assessed using fMRI 6 months after encoding, in normal participants who either had slept or had been totally sleep deprived during the first postencoding night. The set of brain areas recruited during recollection changed over time, regardless of emotional significance of encoded stimuli. After 6 months (compared with $3 \mathrm{~d}$ ), recollection was associated with increased cortical activity, contrasting with the conspicuous absence of significant hippocampal response. These findings support the view that systems consolidation of declarative memories involves a reorganization of memories within cerebral networks, transferring progressively the burden of retention to neocortical, rather than hippocampal, areas. Second, sleep during the initial postencoding night profoundly influences systems-level consolidation of emotional memories and modifies the cortical functional specialization and integration associated with recollection of emotional memories in the long term.

\section{Progressive reorganization of declarative memories, independently of emotion}

The correlates of recollection significantly changed between $3 \mathrm{~d}$ and 6 month retests, regardless of the emotional significance of encoded items. Six months after encoding, responses elicited by recollection were increased in several frontal and parietal cortices. In the hippocampus, the significant responses recorded $3 \mathrm{~d}$ after encoding (Sterpenich et al., 2007) were no longer observed after 6 months. Collectively, these findings are in agreement with a systems-level consolidation of declarative memory characterized by a decreased contribution of mesiotemporal structures and a progressive reorganization within cortical networks, including associative regions (e.g., vMPFC) and sensory areas (e.g., fusiform gyrus). Sleep during the postencoding night only moderately influenced these modifications in responses. No significant changes in recollection-related responses (all valences included) were detected between RS and TSD groups from $3 \mathrm{~d}$ to 6 month retests, although recollection at 6 months elicited significant responses in the vMPFC and the fusiform gyrus in the RS that were not detected in the TSD group (exclusive masking).

Progressive reorganization of declarative memories: influence of emotion and sleep

In contrast, sleep on the first postencoding night selectively enhanced the systems-level consolidation of emotional, relative to
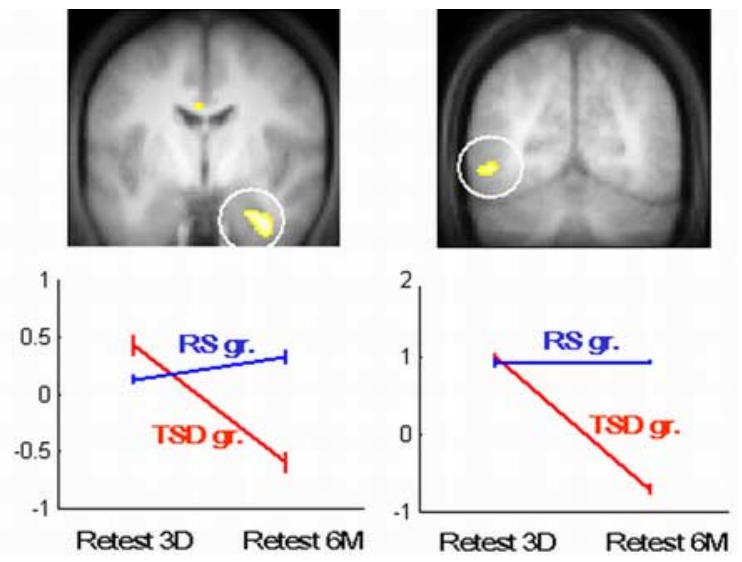

Retest $6 \mathrm{M}$

Figure 5. Effect of sleep on emotional memory, specific to long-term retention. Memory ( $\mathrm{R}>\mathrm{K}$ ) by emotion (Neg $>$ Neu) by sleep status (RS $>$ TSD) by delay ( 6 month retest $>3$ d retest) interaction. From left to right are the following: vMPFC, amygdala, and middle occipital gyrus. Top, Functional results are displayed on the mean structural MR image (display at $p<0.001$, uncorrected). Bottom, Evolution of the activity estimates of negative minus neutral R responses across time (arbitrary units \pm SEM).

neutral memories. Three days after encoding, successful recollection of emotional stimuli elicited larger responses in the hippocampus and the medial prefrontal cortex in the sleep group than in the sleep-deprived group. The hippocampus and medial prefrontal cortex were functionally more connected during recollection of emotional than neutral items, and more so in sleeping than in sleep-deprived subjects (Sterpenich et al., 2007). At 6 months, responses associated with recollection of emotional items in the sleep group were associated with responses in medial frontal and parietal areas, occipital cortices and the amygdala, but no longer in the hippocampus.

The vMPFC detected 6 months after encoding is more posterior and ventral than the area we observed $3 \mathrm{~d}$ after encoding (Sterpenich et al., 2007) but is remarkably similar to the one reported by two previous independent studies of long-term declarative memory (Takashima et al., 2006; Gais et al., 2007). Ventral MPFC has been viewed as an executive area that would index and bind information stored in distributed cortical areas, allowing for retrieval of remote memories (Takashima et al., 2006). An alternative interpretation is that vMPFC (also activated in the contrast of emotion) is related to goal-directed behavior and reward (Heinzel et al., 2005; Lecourtier et al., 2008). This region would respond to successful recollection associated with an internal reward more efficiently when memories have been processed during sleep early on during consolidation.

Two amygdalar clusters significantly responded to recollection 6 months after encoding in RS group. They differ from the medial amygdala identified during recollection $3 \mathrm{~d}$ after encoding in sleep-deprived subjects (supplemental Fig. S1, available at www.jneurosci.org as supplemental material). These findings suggest that various functional stages of memory consolidation involve separate portions of the amygdala.

Data additionally show that responses elicited by recollection 
of emotional material in amygdala and frontal and occipital cortices increased between $3 \mathrm{~d}$ and 6 month after encoding, differently in subjects allowed to sleep, compared with sleep-deprived participants. In addition, the functional connectivity was also significantly enhanced for emotional relative to neutral items and more so in the sleep than the sleep-deprived group between vMPFC and the precuneus, two areas tightly related to memory retrieval (Henson et al., 2005; Cavanna and Trimble, 2006), between the amygdala and the fusiform gyrus, two regions respectively involved in processing emotional and perceptual aspects of visual stimuli (Morris et al., 1998; Adolphs, 2004) and between the amygdala and the vMPFC. Collectively, these results support our hypothesis that off-line processing of emotional memories taking place during sleep on the first posttraining night triggers a consolidation process that progressively develops during the following weeks and results in strengthening connections between the amygdala, the vMPFC, and occipital areas, a network that is recruited during subsequent recollection of remote emotional memories. Although the initially sleep-deprived subjects did sleep as usual during the consolidation period from the second postencoding night onward, the consolidation process seems to follow a divergent trajectory in such a way that brain responses elicited by recollection continue to differ from the sleep group 6 months after encoding. The potential advantage brought about by sleep-dependent consolidation of emotional memories does not appear clearly in the present study, since no difference in emotional memory was observed between groups at 6 months postencoding. These negative behavioral results partly arise from our design, which implied an incident encoding of a large number of items. A positive behavioral effect of sleep on remote retrieval of emotional memories might have been detected with a deeper explicit encoding of a smaller number of items (Wagner et al., 2006) or in conditions of interference (Ellenbogen et al., 2006).

\section{Alternative interpretations}

The emotionality of the items might have changed over time on an individual basis and the emotion elicited by intrinsically emotional stimuli might therefore have influenced memory retrieval. In support of this hypothesis, pupillary size, taken as a measure of the arousal elicited by stimuli, changed from encoding to early and late retests. However, the time course of pupillary changes did not differ between groups and is thus not likely to account for the differences in brain responses observed between the sleep and sleep-deprived group.

Another possibility is that retrieval effort increases as retest delay lengthens, explaining larger responses at 6 months than $3 \mathrm{~d}$. Again, one would have to assume that the retrieval effort differs between sleep and sleep-deprived groups to account for the between-group differences reported above.

Moreover, subjects are reexposed to the learned material during the retrieval session after $3 \mathrm{~d}$. Although they do not receive any feedback concerning the accuracy of their responses, the second exposure can potentially lead to memory reactivation and reconsolidation and thus influences the retrieval session after 6 months. However, this process occurs in both groups of subjects and its effect is minimized in the between-group comparisons.

\section{Cellular mechanisms of memory consolidation during sleep}

Our results shed no light on the mechanisms that underpin offline memory processing during sleep. At present, two main hypotheses are proposed. For some authors (Tononi and Cirelli, 2003), learning would result in a net increase in synaptic strength in many brain circuits, a condition that is not energetically sustainable in the long term. The role of sleep, especially of the cellular activity associated with slow waves, would be to downscale synaptic strength to a baseline level, a mechanism that would eventually consolidate memory. Although these mechanisms potentially participated in consolidating engrams in regions involved in encoding (e.g., occipital areas and amygdala for emotional items), they would not easily account for the recruitment of novel areas during long-term retrieval, for instance the vMPFC. Indeed, we could not detect any significant responses in the latter areas during neither encoding nor early retrieval, a finding that speaks against an initial local synaptic potentiation.

Another hypothesis proposes a coordinated replay of the neural pattern between hippocampal and neocortical areas during sleep (Maquet, 2001; Stickgold et al., 2001), leading to the gradual memory storage within distributed cortical networks. Evidence for such sequential replay has been obtained by multiunit recordings in rodents, from the hippocampus (Nádasdy et al., 1999), sensory cortices (Ji and Wilson, 2007), and the MPFC (Euston et al., 2007). The present data fit well with the spatial reorganization of memories in cortical areas implied by this model.

\section{Are there any selective mechanism for the off-line processing of emotional memories during sleep?}

Emotional memory is especially sensitive to REM sleep deprivation, suggesting that REM sleep is particularly involved in the consolidation this type of memory (Wagner et al., 2001; Silvestri, 2005). It is usually thought that a modulation of hippocampal formation or of hippocampo-neocortical interactions by the amygdala, a structure highly active during REM sleep (Maquet et al., 1996), might account for this effect. Future research is needed to characterize the off-line processing of emotional memory during sleep.

\section{References}

Adolphs R (2004) Emotional vision. Nat Neurosci 7:1167-1168.

Alvarez P, Squire LR (1994) Memory consolidation and the medial temporal lobe: a simple network model. Proc Natl Acad Sci U S A 91:7041-7045.

Beck AT, Epstein N, Brown G, Steer RA (1988) An inventory for measuring clinical anxiety: psychometric properties. J Consult Clin Psychol 56:893-897.

Bermond B, Vorst HC, Vingerhoets AJ, Gerritsen W (1999) The Amsterdam Alexithymia Scale: its psychometric values and correlations with other personality traits. Psychother Psychosom 68:241-251.

Born J, Rasch B, Gais S (2006) Sleep to remember. Neuroscientist 12:410-424.

Buysse DJ, Reynolds CF 3rd, Monk TH, Berman SR, Kupfer DJ (1989) The Pittsburgh Sleep Quality Index: a new instrument for psychiatric practice and research. Psychiatry Res 28:193-213.

Cavanna AE, Trimble MR (2006) The precuneus: a review of its functional anatomy and behavioural correlates. Brain 129:564-583.

Daselaar SM, Fleck MS, Cabeza R (2006) Triple dissociation in the medial temporal lobes: recollection, familiarity, and novelty. J Neurophysiol 96:1902-1911.

Diana RA, Yonelinas AP, Ranganath C (2007) Imaging recollection and familiarity in the medial temporal lobe: a three-component model. Trends Cogn Sci 11:379-386.

Dolcos F, LaBar KS, Cabeza R (2005) Remembering one year later: role of the amygdala and the medial temporal lobe memory system in retrieving emotional memories. Proc Natl Acad Sci U S A 102:2626-2631.

Dudai Y (2004) The neurobiology of consolidations, or, how stable is the engram? Annu Rev Psychol 55:51-86.

Ellenbogen JM, Hulbert JC, Stickgold R, Dinges DF, Thompson-Schill SL (2006) Interfering with theories of sleep and memory: sleep, declarative memory, and associative interference. Curr Biol 16:1290-1294.

Euston DR, Tatsuno M, McNaughton BL (2007) Fast-forward playback of recent memory sequences in prefrontal cortex during sleep. Science 318:1147-1150. 
Fletcher PC, Frith CD, Rugg MD (1997) The functional neuroanatomy of episodic memory. Trends Neurosci 20:213-218.

Frankland PW, Bontempi B (2005) The organization of recent and remote memories. Nat Rev Neurosci 6:119-130.

Friston KJ, Penny W (2003) Posterior probability maps and SPMs. Neuroimage 19:1240-1249.

Gais S, Albouy G, Boly M, Dang-Vu TT, Darsaud A, Desseilles M, Rauchs G, Schabus M, Sterpenich V, Vandewalle G, Maquet P, Peigneux P (2007) Sleep transforms the cerebral trace of declarative memories. Proc Natl Acad Sci U S A 104:18778-18783.

Gitelman DR, Penny WD, Ashburner J, Friston KJ (2003) Modeling regional and psychophysiologic interactions in fMRI: the importance of hemodynamic deconvolution. Neuroimage 19:200-207.

Heinzel A, Bermpohl F, Niese R, Pfennig A, Pascual-Leone A, Schlaug G, Northoff G (2005) How do we modulate our emotions? Parametric fMRI reveals cortical midline structures as regions specifically involved in the processing of emotional valences. Brain Res Cogn Brain Res 25:348-358.

Henson RN, Hornberger M, Rugg MD (2005) Further dissociating the processes involved in recognition memory: an FMRI study. J Cogn Neurosci 17:1058-1073.

Horne JA, Ostberg O (1976) A self-assessment questionnaire to determine morningness-eveningness in human circadian rhythms. Int J Chronobiol 4:97-110.

Hu P, Stylos-Allan M, Walker MP (2006) Sleep facilitates consolidation of emotional declarative memory. Psychol Sci 17:891-898.

Ji D, Wilson MA (2007) Coordinated memory replay in the visual cortex and hippocampus during sleep. Nat Neurosci 10:100-107.

Johns MW (1991) A new method for measuring daytime sleepiness: the Epworth sleepiness scale. Sleep 14:540-545.

Lane RD, Chua PM, Dolan RJ (1999) Common effects of emotional valence, arousal and attention on neural activation during visual processing of pictures. Neuropsychologia 37:989-997.

Lang PJ, Bradley MM, Cuthbert BN (1999) International Affective Picture System (IAPS): instruction manual and affective ratings. Gainesville, FL: The Center for Research in Psychophysiology, University of Florida.

Lecourtier L, Defrancesco A, Moghaddam B (2008) Differential tonic influence of lateral habenula on prefrontal cortex and nucleus accumbens dopamine release. Eur J Neurosci 27:1755-1762.

Maquet P (2001) The role of sleep in learning and memory. Science 294:1048-1052.

Maquet P, Péters J, Aerts J, Delfiore G, Degueldre C, Luxen A, Franck G (1996) Functional neuroanatomy of human rapid-eye-movement sleep and dreaming. Nature 383:163-166.

Morris JS, Friston KJ, Büchel C, Frith CD, Young AW, Calder AJ, Dolan RJ
(1998) A neuromodulatory role for the human amygdala in processing emotional facial expressions. Brain 121:47-57.

Müller G, Pilzecker A (1900) Experimentelle beitrage zur lehre von Gedächtnis. Z Psychol 1:1-300.

Nádasdy Z, Hirase H, Czurkó A, Csicsvari J, Buzsáki G (1999) Replay and time compression of recurring spike sequences in the hippocampus. J Neurosci 19:9497-9507.

Nishida M, Pearsall J, Buckner RL, Walker MP (2009) REM sleep, prefrontal theta, and the consolidation of human emotional memory. Cereb Cortex 19:1158-1166.

Oldfield RC (1971) The assessment and analysis of handedness: the Edinburgh inventory. Neuropsychologia 9:97-113.

Payne JD, Stickgold R, Swanberg K, Kensinger EA (2008) Sleep preferentially enhances memory for emotional components of scenes. Psychol Sci 19:781-788.

Pessoa L, McKenna M, Gutierrez E, Ungerleider LG (2002) Neural processing of emotional faces requires attention. Proc Natl Acad Sci U S A 99:11458-11463.

Silvestri AJ (2005) REM sleep deprivation affects extinction of cued but not contextual fear conditioning. Physiol Behav 84:343-349.

Snodgrass JG, Corwin J (1988) Pragmatics of measuring recognition memory: applications to dementia and amnesia. J Exp Psychol Gen 117:34-50.

Steer RA, Ball R, Ranieri WF, Beck AT (1997) Further evidence for the construct validity of the Beck Depression Inventory-II with psychiatric outpatients. Psychol Rep 80:443-446.

Sterpenich V, Albouy G, Boly M, Vandewalle G, Darsaud A, Balteau E, Dang-Vu TT, Desseilles M, D'Argembeau A, Gais S, Rauchs G, Schabus M, Degueldre C, Luxen A, Collette F, Maquet P (2007) Sleep-related hippocampo-cortical interplay during emotional memory recollection. PLoS Biol 5:e282.

Stickgold R, Hobson JA, Fosse R, Fosse M (2001) Sleep, learning, and dreams: off-line memory reprocessing. Science 294:1052-1057.

Takashima A, Petersson KM, Rutters F, Tendolkar I, Jensen O, Zwarts MJ, McNaughton BL, Fernandez G (2006) Declarative memory consolidation in humans: a prospective functional magnetic resonance imaging study. Proc Natl Acad Sci U S A 103:756-761.

Tononi G, Cirelli C (2003) Sleep and synaptic homeostasis: a hypothesis. Brain Res Bull 62:143-150.

Vuilleumier P, Armony JL, Driver J, Dolan RJ (2003) Distinct spatial frequency sensitivities for processing faces and emotional expressions. Nat Neurosci 6:624-631.

Wagner U, Gais S, Born J (2001) Emotional memory formation is enhanced across sleep intervals with high amounts of rapid eye movement sleep. Learn Mem 8:112-119.

Wagner U, Hallschmid M, Rasch B, Born J (2006) Brief sleep after learning keeps emotional memories alive for years. Biol Psychiatry 60:788-790. 